\title{
ATRX and DAXX: Mechanisms and Mutations
}

\author{
Michael A. Dyer, ${ }^{1}$ Zulekha A. Qadeer, ${ }^{2,3}$ David Valle-Garcia, ${ }^{2}$ and Emily Bernstein ${ }^{2,3}$ \\ ${ }^{1}$ Department of Developmental Neurobiology, St. Jude Children's Research Hospital, Memphis, \\ Tennessee 38105 \\ ${ }^{2}$ Departments of Oncological Sciences and Dermatology, Icahn School of Medicine at Mount Sinai, \\ New York, New York 10029 \\ ${ }^{3}$ Graduate School of Biomedical Sciences, Icahn School of Medicine at Mount Sinai, New York, \\ New York 10029 \\ Correspondence: michael.dyer@stjude.org; emily.bernstein@mssm.edu
}

Recent genome sequencing efforts in a variety of cancers have revealed mutations and/or structural alterations in ATRX and DAXX, which together encode a complex that deposits histone variant $\mathrm{H} 3.3$ into repetitive heterochromatin. These regions include retrotransposons, pericentric heterochromatin, and telomeres, the latter of which show deregulation in ATRX/DAXX-mutant tumors. Interestingly, ATRX and DAXX mutations are often found in pediatric tumors, suggesting a particular developmental context in which these mutations drive disease. Here we review the functions of ATRX and DAXX in chromatin regulation as well as their potential contributions to tumorigenesis. We place emphasis on the chromatin remodeler ATRX, which is mutated in the developmental disorder for which it is named, $\alpha$-thalassemia, mental retardation, $\mathrm{X}$-linked syndrome, and at high frequency in a number of adult and pediatric tumors.

$\mathrm{R}^{\mathrm{e}}$ ecent whole-genome and exome sequencing efforts across a wide spectrum of cancers have unexpectedly revealed chromatin remodeling-encoding genes as being frequently altered (Jiao et al. 2011; Schwartzentruber et al. 2012; Cheung and Dyer 2013; Helming et al. 2014). The ATRX gene, which encodes a SWI/SNFlike chromatin remodeling protein, is frequently mutated in a variety of tumors, including adult lower-grade gliomas, pediatric glioblastoma multiforme, pediatric adrenocortical carcinoma, osteosarcoma, and neuroblastoma (Table 1). Some, but not all, of these cancers also harbor DAXX and/or H3F3A (H3.3) mutations (Schwartzentruber et al. 2012; Wu et al. 2012).
Given that ATRX and DAXX form a histone chaperone complex that deposits histone variant H3.3 into specific genomic regions, it is intriguing that mutations in the histone as well as its chaperone complex have been identified (Schwartzentruber et al. 2012). Although certain H3.3 mutations appear to be associated with altered Polycomb repressive complex 2 (PRC2) activity (e.g., H3.3K27M) (Bender et al. 2013; Chan et al. 2013; Lewis et al. 2013; Funato et al. 2014), the mechanisms by which ATRX and $D A X X$ mutations promote oncogenesis may be distinct from those elicited by $\mathrm{H} 3.3$ mutation.

Given the identification of ATRX and DAXX mutations in cancer, these factors have received

Editors: Scott A. Armstrong, Steven Henikoff, and Christopher R. Vakoc

Additional Perspectives on Chromatin Deregulation in Cancer available at www.perspectivesinmedicine.org

Copyright (C) 2017 Cold Spring Harbor Laboratory Press; all rights reserved; doi: 10.1101/cshperspect.a026567 Cite this article as Cold Spring Harb Perspect Med 2017;7:a026567 
M.A. Dyer et al.
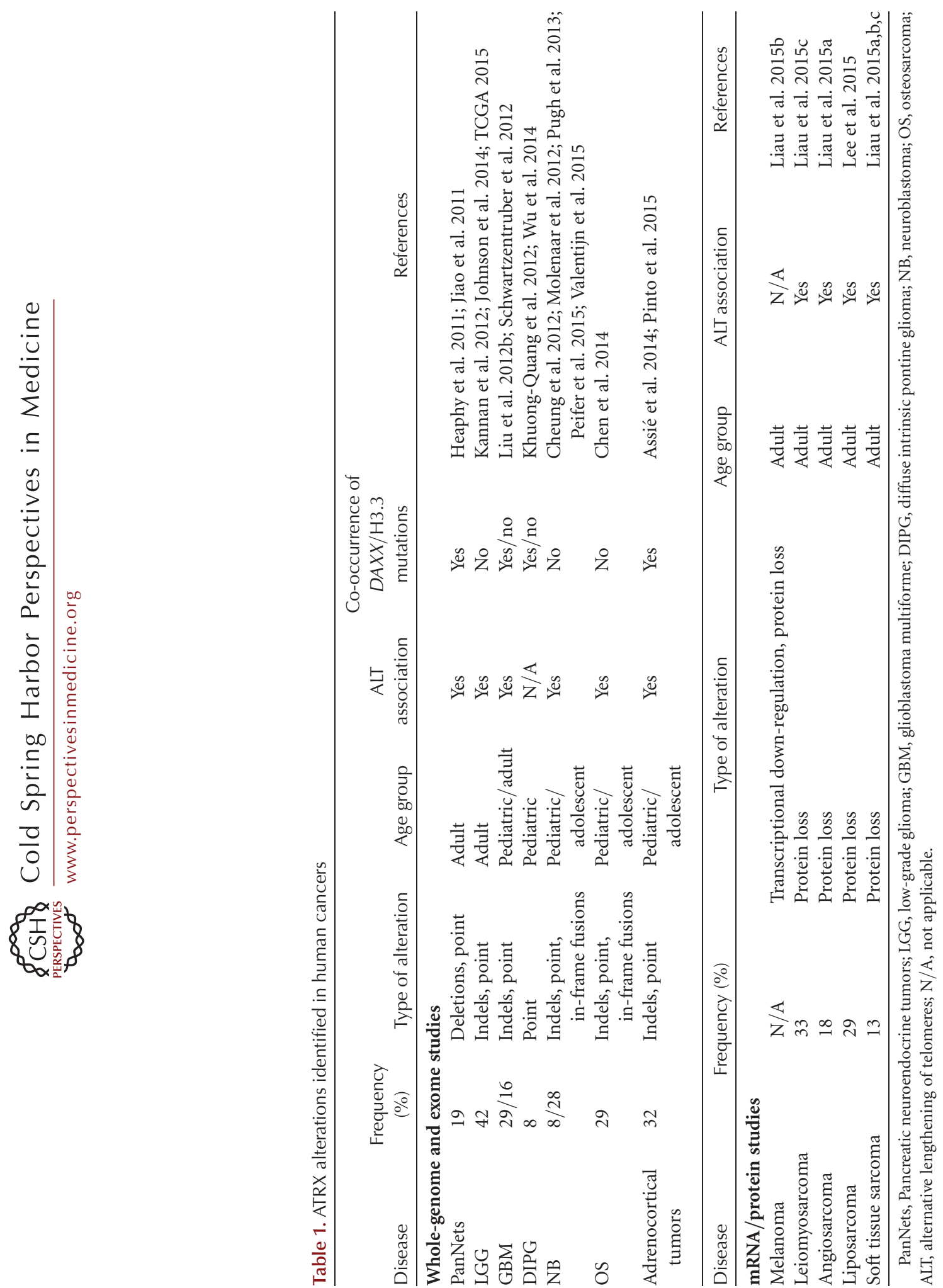
renewed attention, particularly in the field of pediatric oncology. To date, however, their role in promoting pathogenesis in both adult and pediatric tumors remains unclear. Here we review the functions of the chromatin remodeler ATRX and the histone chaperone DAXX in the context of cellular biology, development, and disease.

\section{ATRX SYNDROME}

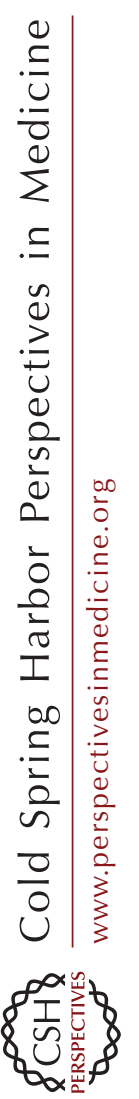

ATRX was first discovered through efforts to identify the genetic lesion that contributes to the $\alpha$-thalassemia, mental retardation, X-linked (ATRX) syndrome (Gibbons et al. 2003). Patients are characterized during early childhood by distinctive craniofacial features, significant developmental delays, genital anomalies and sterility, microcephaly, and severe intellectual disabilities (Gibbons et al. 2012). Coupled to these features, patients with ATRX syndrome also present with varying degrees of anemia caused by $\alpha$-thalassemia, a condition caused by deficient $\alpha$-globin expression (Gibbons et al. 2008). Consistent with a recessive X-linked inheritance pattern, ATRX syndrome is predominant in males. However, there are rare reports of ATRX syndrome in 46XX heterozygous females, which show an uneven pattern of Xinactivation that favors the expression of the mutant allele (Badens et al. 2006b).

Importantly, mutations in ATRX are the only genetic lesions that cause ATRX syndrome (Gibbons et al. 2008). This suggests that ATRX is essential for diverse developmental processes across the ectodermal (neural crest and central nervous system [CNS]), mesodermal (bone and erythroid lineage), and endodermal (genitalia) lineages. Interestingly, mutations in genes that encode proteins that interact with ATRX, such as DAXX, have not been identified in patients with ATRX syndrome. Moreover, ATRX patients do not have an increased incidence of cancer. Taken together, ATRX plays a unique role in development and raises questions about tissue specificity, as well as its role in promoting cancer.

Considering the monogenic origins of ATRX syndrome, it is important to identify the type of mutations involved and their clinical severity. For example, mutations in the amino-terminal ADD (ATRX-DNTM3-DNMT3L) domain produce more severe psychomotor phenotypes than do mutations in the carboxyterminal helicase domain (Badens et al. 2006a). Interestingly, ATRX syndrome mutations almost exclusively lie in either of these two domains (Fig. 1A). ATRX's helicase domain has DNA translocase activity via ATP-dependent hydrolysis (Xue et al. 2003; Mitson et al. 2011). In fact, inactivating mutations in this domain results in translocase defects, and one particular disease-causing mutation even uncouples ATP hydrolysis from DNA binding (Mitson et al. 2011). The ADD domain contains a GATA-like domain and a PHD (plant homeodomain) finger, which together recognize $\mathrm{H} 3 \mathrm{~K} 9 \mathrm{me} 3$ when unmethylated at $\mathrm{H} 3 \mathrm{~K} 4$ (Dhayalan et al. 2011; Eustermann et al. 2011; Iwase et al. 2011). Interestingly, a nonsense mutation at residue 37 of ATRX is associated with a milder phenotype than that associated with missense mutations in the ADD and helicase domains (Guerrini et al. 2000). The nonsense mutation at residue 37 is spliced out of a subset of transcripts, which partially restores ATRX protein function. Indeed, it has been proposed that virtually all mutations in patients with ATRX syndrome represent hypomorphs caused by protein destabilizing effects of the mutations (Gibbons et al. 2008). Because mutations in numerous cancers occur outside of the ADD and helicase domains and span widely across the ATRX coding region (Fig. 1), they have been proposed to be loss-of-function mutations (Watson et al. 2015). Consequently, ATRX syndrome mutations may shed light on our understanding of somatic ATRX mutations in cancer.

\section{MOUSE MODELS OF ATRX DEFICIENCY}

Consistent with the hypothesis that ATRX is required for embryonic development, Atrxnull mice die at E9.5 (Garrick et al. 2006). Moreover, conditional inactivation of Atrx in the developing forebrain results in perinatal lethality. The frontal cortex, subiculum, and hippocampus are reduced in size, and the dentate 
M.A. Dyer et al.

A
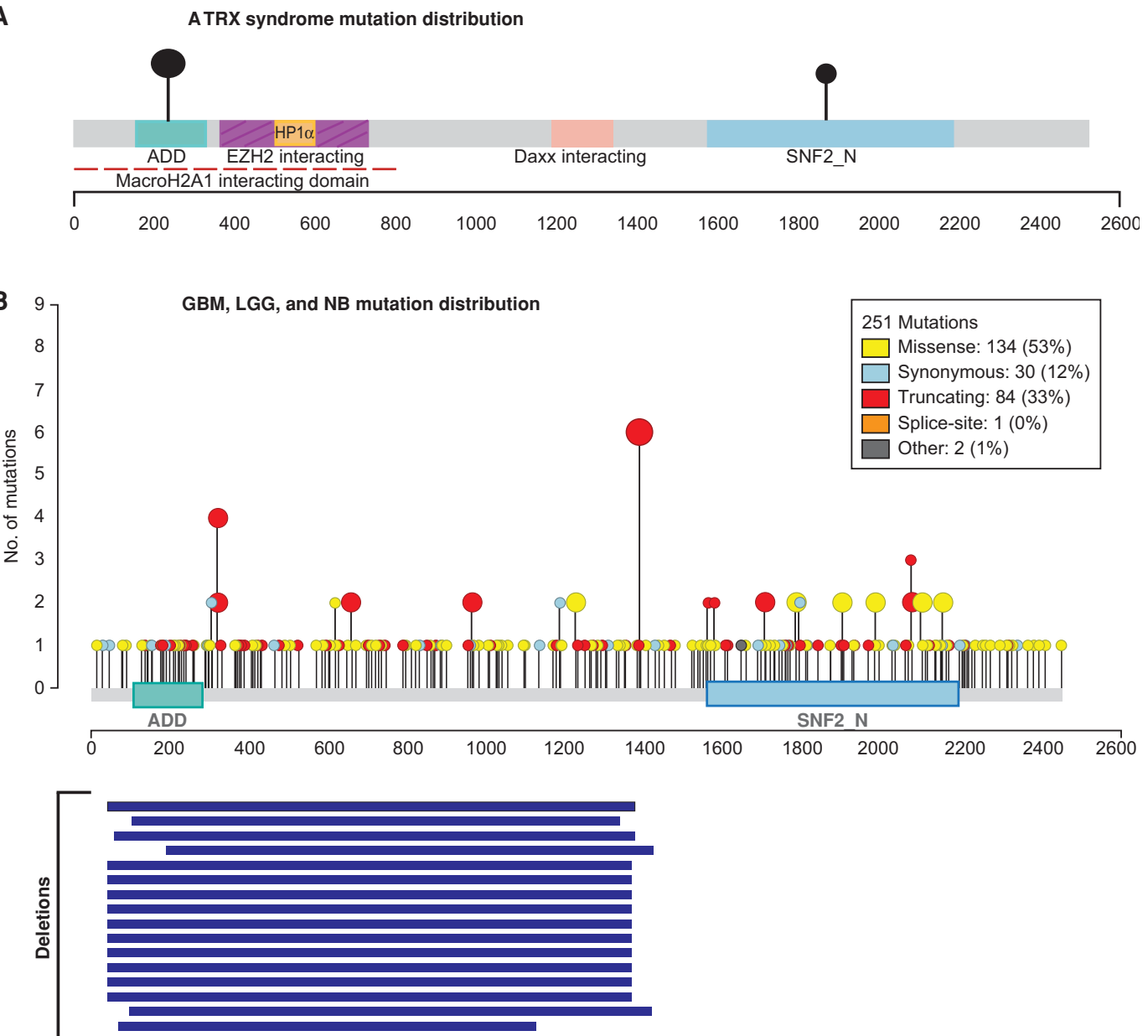

Figure 1. Mutations identified in ATRX syndrome and a subset of cancers. (A) Schematic summarizing that the mutations identified in patients with ATRX syndrome are concentrated in the ADD and SNF2_N helicase domains. Mutations are represented by a black needle; larger needle head size and height indicate larger mutation frequency. $(B)$ Needle plot depicting the distribution of mutations identified in GBM (glioblastoma multiforme), LGG (low-grade glioma), and NB (neuroblastoma) along the ATRX protein product (GonzalezPerez et al. 2013). The needles' head size and height indicate mutational recurrence. Note that these mutations are present throughout the gene locus and not concentrated at one particular domain. Blue bars below protein product represent in-frame fusions identified in NB (Cheung et al. 2012).

gyrus is completely absent in Atrx ${ }^{\text {Lox }}$;Foxg1-Cre mice (Bérubé et al. 2005). There is no defect in neural progenitor cell proliferation, but cells undergo apoptosis as they exit the cell cycle and initiate their differentiation programs. This effect appears to be cell autonomous. Thus, the phenotype in mice is much more severe than that in humans, because patients with ATRX mutations can survive to adulthood.
These findings in genetically engineered mouse models are consistent with the hypothesis that almost all ATRX alleles in patients with ATRX syndrome represent hypomorphs (Gibbons et al. 2008). To date, hypomorphic alleles of Atrx have not been modeled in mice.

There are additional cell-type-specific defects in development upon Atrx inactivation. For example, the amacrine and horizontal inter- 
ATRX/DAXX in Cancer

neurons are lost in Atrx-deficient retinae (Medina et al. 2009). Up to $23 \%$ of patients with ATRX syndrome have visual anomalies (Medina et al. 2009), but whether these are attributable to defects in interneuron differentiation and/or survival remains unknown. The consequence of Atrx inactivation in skeletal muscle results in defective regenerative capacity of satellite cells (Huh et al. 2012). Skeletal muscle homeostasis requires continuous production of myoblasts from satellite cells, which then differentiate and form myotubes. Myoblasts derived from Atrx-deficient satellite cells are defective in cell cycle progression and show evidence of DNA damage. The proliferating myoblasts have elevated levels of both phosphorylated ATM and Chk1, which is indicative of replicative stress, and this effect is cell autonomous. In addition, the telomeres are unstable in Atrx-deficient myoblasts and show increased rates of merging, duplication, and bridging (Huh et al. 2012). These findings are consistent with the reported roles of ATRX in human cells (see below).

Collectively, these studies highlight the major mechanisms underlying developmental defects that result from Atrx inactivation during development. Specifically, they underscore the lineage-specific defects in differentiation (CNS) and in progenitor cell proliferation as a result of replicative stress-induced DNA damage (muscle). Similar defects have been reported in other cell types and tissues, such as embryonic stem cells, macrophages, Sertoli cells, and limb mesenchyme (Bagheri-Fam et al. 2011; Conte et al. 2012; Solomon et al. 2013; Clynes et al. 2014). It is possible that in lineages such as muscle wherein there is massive proliferative expansion through a stem-cell-related mechanism (i.e., satellite cells), the DNA replication phenotype is predominant. However, in the developing CNS wherein proliferation during development is primarily limited to multipotent progenitor cells, replicative stress might not be as important as lineage-specific defects during differentiation. Thus, the varied roles of Atrx in differentiation can be a starting point to explain the phenotypes across mesodermal, endodermal, and ectodermal lineages. Moreover, it can provide a context for understanding the role of ATRX in cancer.

\section{DEATH DOMAIN-ASSOCIATED PROTEIN DAXX}

DAXX was originally identified as a Fas death receptor binding protein that induced apoptosis via JNK pathway activation (Yang et al. 1997). Thus, it was coined the death domainassociated protein, DAXX. However, its role in apoptosis remains unclear because of conflicting reports, including the fact that the knockout mouse resulted in extensive apoptosis and embryonic lethality, rather than the expected antiapoptotic phenotype (Michaelson et al. 1999). Moreover, DAXX was found to be primarily a nuclear protein in which it localizes to promyelocytic leukemia (PML) bodies and was suggested to promote sensitivity to the Fas receptor from its nuclear location (Torii et al. 1999; Zhong et al. 2000). DAXX was also reported to interact with a series of transcription factors (TFs) and histone deacetylase (HDAC) complexes (Hollenbach et al. 1999, 2002; Li et al. 2000). Thus, by the early 2000s, the primary role proposed for DAXX function was in regulating transcription, although the mechanism by which it did so was unclear.

Although ATRX function was studied in the context of ATRX syndrome and DAXX function in the context of apoptosis, two independent biochemical studies showed that ATRX and DAXX are in fact components of a distinct nuclear complex (Xue et al. 2003; Tang et al. 2004). It was not until the purification of histone variant chaperone complexes some years later by the groups of Hamiche and Allis that the function of the ATRX/DAXX complex would be assigned as an H3.3-specfic deposition complex (see below) (Goldberg et al. 2010; Drane et al. 2010; Lewis et al. 2010). Here, we outline the molecular mechanisms of ATRX and DAXX function as a complex as well as their independent functions in chromatin.

\section{ATRX AND DAXX REGULATE HISTONE VARIANT DEPOSITION}

Biochemical investigation of $\mathrm{H} 3.3$ chaperone complexes identified ATRX and DAXX, and found this complex to be necessary for H3.3 
M.A. Dyer et al.

deposition at telomeres (Goldberg et al. 2010; Lewis et al. 2010) and pericentric heterochromatin (Drane et al. 2010) (Fig. 2A). Interestingly, these studies identified a second histone chaperone complex for H3.3, as the HIRA complex had been previously identified to deposit $\mathrm{H} 3.3$ at euchromatic regions of the genome, such as transcriptional start sites and gene bodies (Ahmad and Henikoff 2002; Tagami et al. 2004). The studies that identified the role of the ATRX/ DAXX complex in H3.3 deposition not only revealed that distinct factors are responsible for H3.3 incorporation at specific genomic locations but also uncovered an unexpected role for H3.3 at heterochromatic regions of the genome.

Within the ATRX/DAXX complex, DAXX was shown as the component containing H3.3 histone chaperone activity (Drane et al. 2010; Lewis et al. 2010). For example, DAXX binds directly to $\mathrm{H} 3.3$ and contributes to the deposition of H3.3-H4 tetramers onto naked DNA (Drane et al. 2010; Lewis et al. 2010). Elegant structural studies resolved DAXX in complex with an $\mathrm{H} 3.3 / \mathrm{H} 4$ dimer and showed that residue G90 (unique to H3.3 vs. canonical H3), is critical for this interaction (Elsässer et al. 2012; Liu et al. 2012a). Consistent with its role as a chromatin remodeler, ATRX binds to DAXX to incorporate $\mathrm{H} 3.3$ into telomeric, pericentromeric, and other repetitive DNA (Fig. 2A) (Drane et al. 2010; Goldberg et al. 2010; Wong 2010). Although the precise role of ATRX in this process remains unclear, it is well established that ATRX can recognize $\mathrm{H} 3 \mathrm{~K} 9 \mathrm{me} 3$ through its $\mathrm{ADD}$ domain and is likely responsible for the recruitment of its binding partner DAXX to such regions.

Before studies implicating ATRX as a component of an H3.3 histone chaperone complex, its role in transcription was studied primarily at the $\alpha$-globin gene cluster in erythroid cells because of the $\alpha$-thalassemia phenotype observed in ATRX syndrome patients. In this particular case, ATRX promotes expression of the $H B A$ genes at the $\alpha$-globin locus on the subtelomere of chromosome 16 (Law et al. 2010). Our group identified a role for the transcriptionally repressive $\mathrm{H} 2 \mathrm{~A}$ variant macroH2A at the $\alpha$-globin lo- cus and telomeres (Fig. 2A). In the absence of ATRX, macroH2A is found enriched at these regions, concomitant with reduced $\alpha$-globin expression-a prevalent feature of ATRX syndrome patients. Interestingly, this function for ATRX in negatively regulating macroH2A deposition at the $\alpha$-globin locus is DAXX-independent (Ratnakumar et al. 2012).

\section{ATRX BINDS TO REPETITIVE REGIONS OF THE GENOME}

Initial immunofluorescence studies of ATRX found it to be localized to condensed DAPIdense regions, suggesting that ATRX is primarily associated with heterochromatin (McDowell et al. 1999; Bérubé et al. 2000; Xue et al. 2003). More recently, ChIP-seq studies identified ATRX enrichment at telomeric, subtelomeric, and pericentric repeats, consistent with the functional studies described above (Law et al. 2010). Additional ChIP-seq analyses found that ATRX is also enriched at the silenced allele of imprinted regions, particular families of retrotransposons, a subset of Grich intragenic regions, and the $3^{\prime}$ exons of genes belonging to the $\mathrm{C} 2 \mathrm{H} 2$ Zinc Finger (ZNFs) family of transcription factors (Elsässer et al. 2015; He et al. 2015; Levy et al. 2015; Sadic et al. 2015; Voon et al. 2015; Valle-Garcia et al. 2016). Some of these recent findings are outlined below.

\section{Telomeres}

The ATRX/DAXX complex is enriched at telomeric repeats where it has been shown to regulate H3.3 deposition (Goldberg et al. 2010; Law et al. 2010; Lewis et al. 2010; Wong 2010) (Fig. 2A). Although the precise function of H3.3 deposition at telomeres remains unclear, ATRX depletion leads to telomere dysfunction (Wong 2010; Watson et al. 2013; Ramamoorthy and Smith 2015; Watson et al. 2015). For example, ATRX loss leads to increased DNA damage, increased rates of telomeric end fusions, persistent telomere cohesion, and general genomic instability (Wong 2010; Watson et al. 2013, 2015; Ramamoorthy 
ATRX/DAXX in Cancer
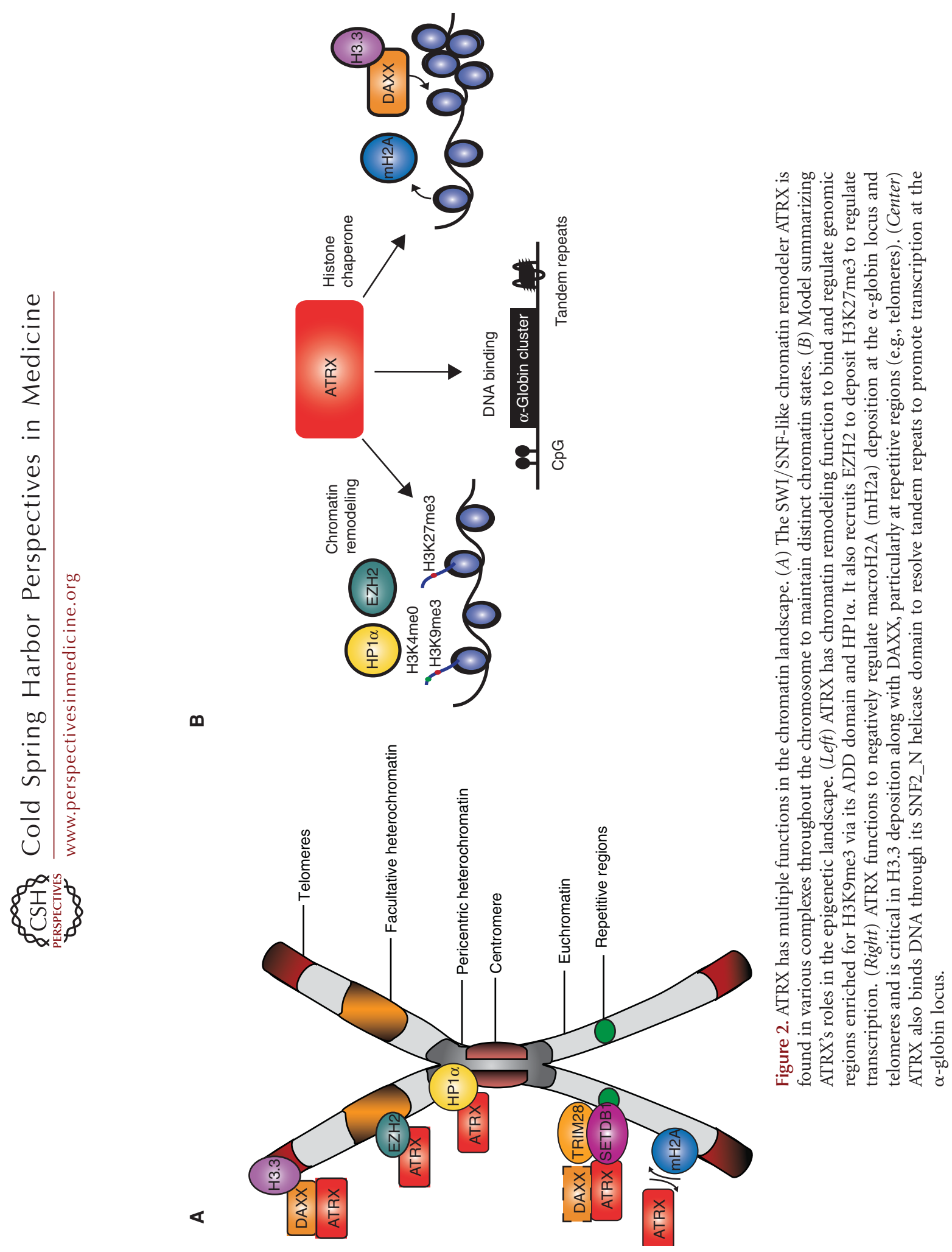
M.A. Dyer et al.

and Smith 2015). Intriguingly, alternative lengthening of telomeres (ALT) is a frequent feature of ATRX-mutated cancers (see below), suggesting that ATRX indeed has an important role in the regulation of telomeres.

\section{G4 Structures}

ATRX syndrome patients display varying degrees of severity of $\alpha$-thalassemia (Gibbons 2006). Chromatin immunoprecipitation followed by high-throughput sequencing (ChIPseq), revealed that ATRX directly binds to Grich tandem repeats upstream of the $\alpha$-globin genes (Fig. 2B) (Law et al. 2010). These repeats tend to form G-quadruplexes (G4) structuressecondary DNA structures that can lead to replicative stress (Huh et al. 2012, Leung et al. 2013, Clynes et al. 2014) or block transcriptional processes (Levy et al. 2015). Such structures are typically found in tandem repeats at telomeres and pericentromeric repeats, but not restricted to them (e.g., intragenic CpGs, differentially methylated regions, etc.) (Huppert and Balasubramanian 2005). Using recombinant ATRX protein, in vitro assays showed binding to preformed G4 oligonucleotides, suggesting that this property of ATRX is in fact direct (Law et al. 2010).

As ATRX binds G4 DNA, it has been suggested to resolve these structures (Law et al. 2010; Clynes and Gibbons 2013). Consistent with this, the severity of the $\alpha$-thalassemia phenotype in ATRX syndrome patients directly correlates with VNTR (variable number tandem repeat) size at the $\alpha$-globin locus (Law et al. 2010). In the absence of ATRX, larger VNTR regions form more G4 structures with increased impact on the transcription of the $\alpha$-globin genes. This is corroborated by findings that show the phenotypes associated with ATRX loss are enhanced with compounds that stabilize G4 DNA (Watson et al. 2013; Clynes et al. 2014, 2015). Further, it has been suggested that one of the mechanisms by which ATRX suppresses recombination at telomeric repeats is by resolving G4 structures through the deposition of H3.3 (Clynes and Gibbons 2013; Clynes et al. 2015).

\section{Transposable Elements}

The ATRX/DAXX complex also mediates the deposition of $\mathrm{H} 3.3$ at retrotransposons, particularly those belonging to the endogenous retrovirus (ERV) family (Elsässer et al. 2015; He et al. 2015; Sadic et al. 2015). The corepressor TRIM28 (also known as KAP1) and the H3K9 histone methyltransferase SETDB1 (also known as ESET), colocalize with ATRX at ERVs (Fig. 2A). ATRX-bound retrotransposons have high levels of $\mathrm{H} 3 \mathrm{~K} 9 \mathrm{me} 3$ that are dependent on the ATRX/DAXX complex. Knockdown of either component, or H3.3 itself, reduces the $\mathrm{H} 3 \mathrm{~K} 9 \mathrm{me} 3$ levels at these repetitive regions and induces aberrant transcription (Elsässer et al. 2015; Sadic et al. 2015). Moreover, ATRX/ DAXX-mediated silencing of ERVs is critical for preimplantation embryos during the wave of global DNA demethylation (He et al. 2015). Collectively, these studies highlight the critical role of ATRX in silencing repetitive elements through regulation of $\mathrm{H} 3 \mathrm{~K} 9 \mathrm{me} 3$.

\section{ZNF Genes}

In line with these findings, we identified the $3^{\prime}$ exons of ZNF genes as ATRX targets in human cells (Valle-Garcia et al. 2016). ZNFs are the largest family of TFs in the human genome (Nowick et al. 2011). Although these regions are not considered repetitive per se, $\mathrm{ZNF}$ share high levels of DNA similarity at their $3^{\prime}$ exons, which contain motifs encoding for their zinc finger domains. In fact, ZNF genes can encode up to 40 copies of the zinc finger motif. Unlike other ATRX-regulated regions (i.e., telomeric repeats, retrotransposons, etc.), ZNFs are actively transcribed and are highly enriched in both H3K36me3 and H3K9me3 at their $3^{\prime}$ exons. Interestingly, depletion of ATRX decreases $\mathrm{H} 3 \mathrm{~K} 9 \mathrm{me} 3$ levels at ZNF $3^{\prime}$ exons and other regions presenting an $\mathrm{H} 3 \mathrm{~K} 9 \mathrm{me} 3 / \mathrm{H} 3 \mathrm{~K} 36 \mathrm{me} 3$ atypical chromatin signature. As a consequence, cells with reduced levels of $\mathrm{H} 3 \mathrm{~K} 9 \mathrm{me} 3$ show increased levels of DNA damage, suggesting that ATRX binds to the $3^{\prime}$ exons of ZNFs to maintain their genomic stability through preservation of H3K9me3. 
Overall, a common feature of ATRX targets is the presence of $\mathrm{H} 3 \mathrm{~K} 9 \mathrm{me} 3$. The loss of $\mathrm{H} 3 \mathrm{~K} 9 \mathrm{me} 3$ at repetitive regions, via ATRX lossof-function, appears to promote genomic instability. However, it remains unclear how the loss of ATRX results in reduced $\mathrm{H} 3 \mathrm{~K} 9 \mathrm{me} 3$ levels. Is ATRX required to maintain $\mathrm{H} 3 \mathrm{~K} 9 \mathrm{me} 3$ through binding of its ADD domain? Does such docking of ATRX at H3K9me3-modified chromatin facilitate DAXX-mediated H3.3 deposition? What is the role of $\mathrm{H} 3.3$ in regulating heterochromatin dynamics? Although all evidence to date suggests that increased genomic instability is a driving feature of $A T R X$-mutated cancers, this important area of research requires further investigation.

\section{ADDITIONAL FUNCTIONS OF ATRX}

Before the discovery that ATRX cooperates with DAXX to deposit $\mathrm{H} 3.3$ at $\mathrm{H} 3 \mathrm{~K} 9$ me3-containing repetitive regions, ATRX was reported to interact with various heterochromatin-related proteins. These protein interaction partners include HP1 $\alpha$ and EZH2, among others (Fig. 2) (Cardoso et al. 1998; Lechner et al. 2005). HP1 $\alpha$ was originally identified to interact with ATRX through a yeast two-hybrid screen (Le Douarin et al. 1996) and later shown to colocalize at pericentric heterochromatin (McDowell et al. 1999). Additional recombinant studies identified a variant $\mathrm{PxVxL}$ motif within ATRX that binds to the chromoshadow domain of HP1 $\alpha$ (Lechner et al. 2005). Curiously, this study and others were unable to copurify these two proteins from cellular extracts (Lechner et al. 2005; Rosnoblet et al. 2011). Thus, although ATRX and HP1 $\alpha$ co-occupy similar genomic regions and cooperate in heterochromatin regulation, their interaction may be transient, such as during the recruitment of ATRX to H3K9me3-enriched regions (Eustermann et al. 2011).

A yeast two-hybrid analysis coupled to in vitro binding assays showed that ATRX also binds to EZH2 (Cardoso et al. 1998); however, until recently, this interaction had not been characterized in cells. Sarma et al. (2014) reported that ATRX plays a role in targeting PRC2 (Polycomb repressive complex 2) in mouse embryonic stem cells, particularly to the inactive $\mathrm{X}$ chromosome $(\mathrm{Xi})$. In this case, the ATRX-EZH2 interaction occurs via the Xist RNA, which is necessary to recruit ATRX to the $\mathrm{Xi}$ and facilitate deposition of H3K27me3. As described above, female ATRX syndrome patients can display skewed X-chromosome inactivation (De la Fuente et al. 2011) and, therefore, H3K27me3 deposition at the Xi (and other genomic regions) may be defective in these patients.

How DAXX plays a role in the above processes remains unclear. ATRX may have particular DAXX-independent roles in the cell. As noted above, DAXX mutations have not been identified in patients with ATRX syndrome. Furthermore, there are certain cancers that harbor ATRX mutations without evidence of DAXX mutations (see below). We previously showed that ATRX regulates macroH2A deposition in a DAXX-independent manner as well (Ratnakumar et al. 2012). Therefore, such DAXX-independent roles of ATRX could mediate critical functions that are perturbed in ATRX-mutant cancers.

\section{ATRX/DAXX MUTATIONS IN CANCER}

Recent years have witnessed a myriad of whole genome/exome sequencing studies identifying mutations in $A T R X$, and to a lesser extent $D A X X$, across a spectrum of tumor types (Table 1). The first observations came from pancreatic neuroendocrine tumors (PanNETs), in which DNA sequencing studies uncovered frequent mutations in the chromatin factors MEN1, DAXX, and ATRX (Jiao et al. 2011). Notably, $D A X X$ and ATRX mutations were found to be mutually exclusive, yet were altered in a strikingly high percentage of tumors (43\%). These mutations ranged from point mutations to insertion or deletions of bases (indels) and were not localized to any particular domains of ATRX, suggesting loss-of-function. In accordance, both ATRX and DAXX mutations correlated with loss of protein expression by IHC analysis. Intriguingly, these mutations associated with ALT as evidenced by fluorescence in situ hybridization (Heaphy et al. 2011; Jiao et al. 2011). 
M.A. Dyer et al.

Subsequently, ATRX and DAXX mutations were identified in pediatric glioblasoma multiforme (GBM) (Fig. 1B) (Schwartzentruber et al. 2012). Remarkably, point mutations in $H 3 F 3 A$ leading to critical amino acid substitutions at residues $\mathrm{K} 27$ (K27M) or G34 (G34R/V) of the histone tail were also identified in pediatric GBM and diffuse pontine glioma (DIPG), a rare pediatric glioma of the brainstem (Khuong-Quang et al. 2012; Wu et al. 2012). In contrast to pediatric GBM, DIPGs harbor only H3.3K27M mutations (not G34R/V) and are extremely prevalent $(70 \%-80 \%)$ (KhuongQuang et al. 2012; Wu et al. 2012). ATRX mutations are found far less frequently $(\sim 10 \%)$ in DIPG and tend to occur in older children (Khuong-Quang et al. 2012). In the context of pediatric GBM, mutations in the ATRX-DAXXH3.3 axis occur in $\sim 45 \%$ of patients (Schwartzentruber et al. 2012) and show a strong correlation between ATRX mutations and ALT (Heaphy et al. 2011; Schwartzentruber et al. 2012).

$\mathrm{H} 3 \mathrm{~F} 3 \mathrm{~A}$ mutations in pediatric cancers represent the first high-frequency mutations in a histone variant to be identified (KhuongQuang et al. 2012; Schwartzentruber et al. 2012; Wu et al. 2012). Intriguingly, H3.3K27M mutations were not found to frequently cooccur with ATRX mutations. Several groups showed that the H3.3K27M mutation induces a dramatic global decrease in H3K27me3 levels. This may be because of sequestration and/or inhibition of the PRC2 subunit, EZH2 (Bender et al. 2013; Chan et al. 2013; Lewis et al. 2013; Funato et al. 2014). This is compelling given that ATRX has also been shown to interact with EZH2 (Cardoso et al. 1998; Sarma et al. 2014) and suggests that both ATRX and H3.3K27M mutations could be independently driving Polycomb dysfunction in cancer. In contrast, H3.3G34R/V mutations do co-occur with ATRX mutations, suggesting distinct functions of each in tumors harboring these mutations (Schwartzentruber et al. 2012; Wu et al. 2014). Given the young age of patients diagnosed with pediatric GBM and DIPG, ATRX mutations may be promoting defects in lineage differentiation in neural stem cells during brain development that culminate in aberrant cell growth and tumor formation. This idea is corroborated by severe defects in the developing brain in the Atrx-deficient mouse (Medina et al. 2009) and in ATRX syndrome in which patients present with brain abnormalities and intellectual disabilities (Gibbons et al. 2008).

Studies in adult glioma patient cohorts showed a strong correlation of ATRX mutations with IDH1 and p53 mutations in lower grade gliomas (LGGs) (astrocytomas and oligodendrogliomas) and secondary GBM (Kannan et al. 2012; Liu et al. 2012b). These mutations are mutually exclusive from (1) FUPB1/CIC mutations, genes that regulate cell growth and are frequently mutated in oligodendroglioma, and (2) the chromosome $1 \mathrm{p} / 19 \mathrm{q}$ co-deletion subtype (Olar and Sulman 2015). Moreover, no H3F3A or DAXX mutations have been identified in adult GBM (Table 1). Similar to pediatric GBM, there is a strong correlation between ATRX mutations and ALT status and such mutations appear to be exclusive from TERT promoter mutations (Liu et al. 2012b; Ceccarelli et al. 2016). These collective findings classify ATRX status to a molecular subtype of adult glioma that is highly recurrent with intermediate prognosis (Olar and Sulman 2015). IDH1 mutations promote DNA and histone hypermethylation through production of an oncometabolite 2-hydroxygluterate (2-HG) that inhibits DNA and histone demethylases (Lu et al. 2012; Turcan et al. 2012). In adult recurrent gliomas, ATRX mutations are sustained in the presence of p53 loss to cooperate with mutant IDH1 to remodel the epigenetic landscape. In contrast, Atrx ablation in adult mouse neurons leads to increased DNA damage and p53-mediated apoptosis (Bérubé et al. 2005), suggesting that ATRX loss in cancer requires additional alterations for survival.

Large-scale efforts to identify mutations in additional pediatric tumors have identified $A T R X$ as frequently mutated in neuroblastoma (NB), osteosarcoma (OS), and adrenocortical tumors (Table 1) (Cheung et al. 2012; Assié et al. 2014; Chen et al. 2014; Pinto et al. 2015). Notably, no $H 3 F 3 A$ and few DAXX mutations were detected in these patient populations. In addition to point mutations and indels, these 
studies also identified large deletions near the amino-terminal region of ATRX leading to inframe fusions of the gene product (Fig. 1B). Notably, such in-frame fusions remove the chromatin binding modules of ATRX including the ADD domain, DAXX binding domain, and the macroH2A1-, HP1 $\alpha$-, and EZH2-interacting regions (Fig. 1B). ATRX mutations and in-frame fusions in NB associate with older age at diagnosis $(28 \%$ found in adolescent patients), with poor prognosis (Table 1) (Cheung et al. 2012).

ATRX syndrome patients show craniofacial abnormalities and developmental delays (Gibbons et al. 2008), supporting the notion that ATRX mutations alter proper neural crest cell lineage specification during development. Thus, ATRX in-frame fusions and mutations in tumors in cells of this lineage may occur early in multipotent progenitors. The selective pressure to produce in-frame fusions in $\mathrm{NB}$ and $\mathrm{OS}$ would indicate a possible gain-of-function for these new ATRX gene products to promote a cellular growth advantage in such progenitors, although this remains to be tested.

Along with tumor sequencing efforts, several recent studies highlight the loss of ATRX mRNA and protein levels in multiple tumor types, including melanoma and various sarcomas (Table 1). These studies showed that as tumors become more advanced or invasive, there is a loss of ATRX expression by qPCR and/or IHC analysis (Qadeer et al. 2014; Liau et al. 2015a,b,c; Lee et al. 2015). Many of these studies uncovered a correlation between ATRX loss and ALT and proposed this co-occurrence as a prognostic factor for poor outcome (Lee et al. 2015; Liau et al. 2015a,b,c).

\section{ATRX/DAXX LOSS AND ALT}

The ALT pathway in cancer was first described in 1997 (Bryan et al. 1997). A subsequent somatic cell hybridization study showed that when cells displaying ALT were fused to normal cells, the ALT phenotype was reversed (Perrem et al. 1999). This finding suggests that ALT is caused by depletion of a factor(s) that could be rescued by normal cells. As described above, diverse cancer types with mutations in ATRX and DAXX are associated with ALT. Approximately $80 \%$ of tumors with ALT harbor mutations in ATRX or DAXX, and conversely $70 \%-$ $80 \%$ of cancers with ATRX mutations show ALT (Lovejoy et al. 2012).

The G-rich telomeric repeats can adopt G4 structures, and H3.3 may prevent G4 formation. Consistent with this model, ATRX-mediated suppression of ALT in U-2 OS cells is dependent on DAXX and H3.3 deposition at telomeres (Clynes et al. 2015). Moreover, this suppression is associated with a reduction in replication fork stalling, likely because of limited formation of G4 structures, which are a substrate for the homologous recombination (HR) at telomeres that results in ALT. An independent study suggested that ALT is dependent on sensing of replicative stress by ATR, and that the role of ATRX in recognizing G4 structures and preventing replicative stress is integral to the ALT phenotype in cancer cells lacking ATRX (O'Sullivan et al. 2014). Clynes et al. (2015) have proposed that in the absence of ATRX, DAXX cannot deposit H3.3 at the G-rich repeats found in telomeres, leading to formation of G4 structures, replication fork stalling, and HR of telomeres through the MRE11-RAD50-NBS1 (MRN) complex, which ultimately leads to ALT. The authors also propose that as a secondary level of regulation, ATRX can sequester the MRN complex to prevent it from mediating HR at telomeres (Clynes et al. 2015). In the absence of ATRX, the MRN complex is released to facilitate HR at telomeres. This model is supported by the findings that patients with ATRX syndrome have aberrant chromatin at telomeres and pericentromeric DNA (Clynes and Gibbons 2013; Clynes et al. 2013).

Ramamoorthy and colleagues have proposed an alternative mechanism to explain the contribution of ATRX mutations to the ALT phenotype. The telomeric poly(ADP-ribose) polymerase tankyrase 1 is required for the resolution of sister telomere cohesion during mitosis (Dynek and Smith 2004). In the absence of ATRX, the histone variant macroH2A1.1 negatively regulates tankyrase, thus inhibiting its ability to resolve sister telomere cohesion. 
M.A. Dyer et al.

This, in turn, allows for aberrant telomere recombination between sister chromatids (Ramamoorthy and Smith 2015).

Although evidence points toward ATRX loss of function driving tumorigenicity through aberrant DNA recombination at repetitive elements, there may be additional functional roles for $A T R X$ mutations in cancer. This is supported by the fact that loss of ATRX alone is not sufficient to drive to ALT (Clynes et al. 2015). ATRX loss may promote cancer via transcriptional changes and/or deregulation of the DNA replication machinery, which remains to be tested.

\section{CONCLUDING REMARKS}

Given the high frequency of ATRX and DAXX mutations in cancer, these chromatin regulators likely play a key role in pathogenesis. However, it remains unclear how. Is it through promoting ALT? If loss of ATRX or DAXX alone is insufficient to promote ALT, what other key factors or mutations are required? It also remains to be determined whether histone variant deposition by the ATRX/DAXX complex is related to its tumor suppressive function. Further, we question why some pediatric cancers show mutations in $A T R X, D A X X$, and $H 3 F 3 A$ (e.g., pediatric GBM), whereas others predominantly harbor mutations only in $A T R X$ (e.g., NB and OS) Are these mutations patterns as clear-cut as reported? Or does it relate to their cell of origin? In turn, how does ATRX regulate cell fate decisions during development that go awry in cancer?

Given the diversity of mutations identified in ATRX, we also question if they are all loss-offunction. For example, in-frame deletions of $A T R X$, such as those found in NB and OS, may have the potential to generate new protein products devoid of critical protein interaction domains. Moreover, as complete absence of ATRX is not well tolerated in development (i.e., CNS), we question whether ATRX alterations are truly loss-of-function or hypomorphic? We also query whether ATRX controls recombination of other repetitive sequences in the genome besides telomeres (e.g., ZNFs), and whether such recombination events play a role in tumorigenesis. Finally, can our understanding of ATRX and DAXX function be modeled in development and disease to provide clues of their roles in promoting cancer (i.e., mouse models)? To our knowledge, there are currently no in vivo models mimicking ATRX or DAXX mutations that address these important questions.

Given that ATRX has critical roles in maintenance of repetitive regions (particularly telomeres), as well as cell cycle regulation (Wong 2010; Ramamoorthy and Smith 2015; Watson et al. 2015; Huh et al. 2016), targeting these perturbed pathways could serve as therapeutic strategies for ATRX-mutant cancers. For example, Flynn et al. (2015) recently reported that ATRX null, ALT positive cells are sensitive to inhibition of the ATR kinase, a key regulator of homologous recombination. Corroborating these findings, another study showed that ATRX ablation caused increased stalled fork replication, DNA damage and subsequent hyperactivation of PARP-1 and ATM kinase. Thus, it was proposed that PARP-1 inhibitors might further exacerbate DNA damage and promote cell death in ATRX null cells (Huh et al. 2016). We posit that other strategies might include the targeting of epigenetic factors that work together with ATRX, which may also be deregulated, such as SETDB1 or EZH2. Collectively, we anticipate that addressing the important questions raised in this review will provide critical information for deciphering and treating these devastating cancers.

\section{ACKNOWLEDGMENTS}

Funding is provided by a graduate fellowship from the National Council of Science and Technology (CONACyT) (239663, CVU 257385) to D.V.-G., the National Cancer Institute (NCI) T32-CA078207 to Z.A.Q., the National Institutes of Health (NIH) EY014867, EY018599, and CA168875, Cancer Center Support from the NCI (CA21765), support from the American Lebanese Syrian Associated Charities (ALSAC), and a grant from Alex's Lemonade Stand Foundation for Childhood Cancer to M.A.D., and St. Baldrick's Foundation to E.B. 


\section{REFERENCES}

Ahmad K, Henikoff S. 2002. The histone variant H3.3 marks active chromatin by replication-independent nucleosome assembly. Mol Cell 9: 1191-1200.

Assié G, Letouzé E, Fassnacht M, Jouinot A, Luscap W, Barreau O, Omeiri $\mathrm{H}$, Rodriguez $\mathrm{S}$, Perlemoine $\mathrm{K}$, René-Corail F, et al. 2014. Integrated genomic characterization of adrenocortical carcinoma. Nat Genet $\mathbf{4 6}$ : 607-612.

Badens C, Lacoste C, Philip N, Martini N, Courrier S, Giuliano F, Verloes A, Munnich A, Leheup B, Burglen L, et al. 2006a. Mutations in PHD-like domain of the ATRX gene correlate with severe psychomotor impairment and severe urogenital abnormalities in patients with ATRX syndrome. Clin Genet 70: 57-62.

Badens C, Martini N, Courrier S, DesPortes V, Touraine R, Levy N, Edery P. 2006b. ATRX syndrome in a girl with a heterozygous mutation in the ATRX Zn finger domain and a totally skewed X-inactivation pattern. Am J Med Genet A 140: 2212-2215.

Bagheri-Fam S, Argentaro A, Svingen T, Combes AN, Sinclair AH, Koopman P, Harley VR. 2011. Defective survival of proliferating Sertoli cells and androgen receptor function in a mouse model of the ATR-X syndrome. Hum Mol Genet 20: 2213-2224.

Bender S, Tang Y, Lindroth AM, Hovestadt V, Jones DTW, Kool M, Zapatka M, Northcott PA, Sturm D, Wang W, et al. 2013. Reduced H3K27me3 and DNA hypomethylation are major drivers of gene expression in $\mathrm{K} 27 \mathrm{M} \mathrm{mu}-$ tant pediatric high-grade gliomas. Cancer Cell 24: 660672.

Bérubé NG, Smeenk CA, Picketts DJ. 2000. Cell cycle-dependent phosphorylation of the ATRX protein correlates with changes in nuclear matrix and chromatin association. Hum Mol Genet 9: 539-547.

Bérubé NG, Mangelsdorf M, Jagla M, Vanderluit J, Garrick D, Gibbons RJ, Higgs DR, Slack RS, Picketts DJ. 2005. The chromatin-remodeling protein ATRX is critical for neuronal survival during corticogenesis. JClin Invest 115: 258-267.

Bryan TM, Englezou A, Dalla-Pozza L, Dunham MA, Reddel RR. 1997. Evidence for an alternative mechanism for maintaining telomere length in human tumors and tumor-derived cell lines. Nat Med 3: 1271-1274.

Cardoso C, Timsit S, Villard L, Khrestchatisky M, Fontès M, Colleaux L. 1998. Specific interaction between the XNP/ ATR- $X$ gene product and the SET domain of the human EZH2 protein. Hum Mol Genet 7: 679-684.

Ceccarelli M, Barthel FP, Malta TM, Sabedot TS, Salama SR, Murray BA, Morozova O, Newton Y, Radenbaugh A, Pagnotta SM, et al. 2016. Molecular profiling reveals biologically discrete subsets and pathways of progression in diffuse glioma. Cell 164: 550-563.

Chan K-M, Fang D, Gan H, Hashizume R, Yu C, Schroeder M, Gupta N, Mueller S, James CD, Jenkins R, et al. 2013. The histone $\mathrm{H} 3.3 \mathrm{~K} 27 \mathrm{M}$ mutation in pediatric glioma reprograms H3K27 methylation and gene expression. Genes Dev 27: 985-990.

Chen X, Bahrami A, Pappo A, Easton J, Dalton J, Hedlund E, Ellison D, Shurtleff S, Wu G, Wei L, et al. 2014. Recurrent somatic structural variations contribute to tumorigenesis in pediatric osteosarcoma. Cell Rep 7: 104-112.

Cheung N-KV, Dyer MA. 2013. Neuroblastoma: Developmental biology, cancer genomics and immunotherapy. Nat Rev Cancer 13: 397-411.

Cheung N-KV, Zhang J, Lu C, Parker M, Bahrami A, Tickoo SK, Heguy A, Pappo AS, Federico S, Dalton J, et al. 2012. Association of age at diagnosis and genetic mutations in patients with neuroblastoma. JAMA 307: 1062-1071.

Clynes D, Gibbons RJ. 2013. ATRX and the replication of structured DNA. Curr Opin Genet Dev 23: 289-294.

Clynes D, Higgs DR, Gibbons RJ. 2013. The chromatin remodeller ATRX: A repeat offender in human disease. Trends Biochem Sci 38: 461-466.

Clynes D, Jelinska C, Xella B, Ayyub H, Taylor S, Mitson M, Bachrati CZ, Higgs DR, Gibbons RJ. 2014. ATRX dysfunction induces replication defects in primary mouse cells. PLoS ONE 9: e92915.

Clynes D, Jelinska C, Xella B, Ayyub H, Scott C, Mitson M, Taylor S, Higgs DR, Gibbons RJ. 2015. Suppression of the alternative lengthening of telomere pathway by the chromatin remodelling factor ATRX. Nat Commun 6: 7538.

Conte D, Huh M, Goodall E, Delorme M, Parks RJ, Picketts DJ. 2012. Loss of Atrx sensitizes cells to DNA damaging agents through p53-mediated death pathways. PLoS ONE 7: e52167.

De La Fuente R, Baumann C, Viveiros MM. 2011. Role of ATRX in chromatin structure and function: Implications for chromosome instability and human disease. Reproduction 142: 221-234.

Dhayalan A, Tamas R, Bock I, Tattermusch A, Dimitrova E, Kudithipudi S, Ragozin S, Jeltsch A. 2011. The ATRXADD domain binds to $\mathrm{H} 3$ tail peptides and reads the combined methylation state of K4 and K9. Hum Mol Genet 20: 2195-2203.

Drane P, Ouararhni K, Depaux A, Shuaib M, Hamiche A. 2010. The death-associated protein DAXX is a novel histone chaperone involved in the replication-independent deposition of H3.3. Genes Dev 24: 1253-1265.

Dynek JN, Smith S. 2004. Resolution of sister telomere association is required for progression through mitosis. Science 304: 97-100.

Elsässer SJ, Huang H, Lewis PW, Chin JW, Allis CD, Patel DJ. 2012. DAXX envelops a histone H3.3-H4 dimer for H3.3specific recognition. Nature 491: 560-565.

Elsässer SJ, Noh KM, Diaz N, Allis CD, Banaszynski LA. 2015. Histone H3.3 is required for endogenous retroviral element silencing in embryonic stem cells. Nature 522: 240-244.

Eustermann S, Yang J-C, Law MJ, Amos R, Chapman LM, Jelinska C, Garrick D, Clynes D, Gibbons RJ, Rhodes D, et al. 2011. Combinatorial readout of histone $\mathrm{H} 3$ modifications specifies localization of ATRX to heterochromatin. Nat Struct Mol Biol 18: 777-782.

Flynn RL, Cox KE, Jeitany M, Wakimoto H, Bryll AR, Ganem NJ, Bersani F, Pineda JR, Suvà ML, Benes $\mathrm{CH}$, et al. 2015. Alternative lengthening of telomeres renders cancer cells hypersensitive to ATR inhibitors. Science 347: 273277.

Funato K, Major T, Lewis PW, Allis CD, Tabar V. 2014. Use of human embryonic stem cells to model pediatric gliomas 
M.A. Dyer et al.

with H3.3K27M histone mutation. Science 346: 15291533.

Garrick D, Sharpe JA, Arkell R, Dobbie L, Smith AJH, Wood WG, Higgs DR, Gibbons RJ. 2006. Loss of Atrx affects trophoblast development and the pattern of X-inactivation in extraembryonic tissues. PLoS Genet 2: e58.

Gibbons R. 2006. $\alpha$-Thalassaemia-mental retardation, X linked. Orphanet J Rare Dis 1: 15.

Gibbons RJ. 2012. $\alpha$-Thalassemia, mental retardation, and myelodysplastic syndrome. Cold Spring Harb Perspect Med 2: a011759.

Gibbons RJ, Pellagatti A, Garrick D, Wood WG, Malik N, Ayyub H, Langford C, Boultwood J, Wainscoat JS, Higgs DR. 2003. Identification of acquired somatic mutations in the gene encoding chromatin-remodeling factor ATRX in the $\alpha$-thalassemia myelodysplasia syndrome (ATMDS). Nat Genet 34: 446-449.

Gibbons RJ, Wada T, Fisher CA, Malik N, Mitson MJ, Steensma DP, Fryer A, Goudie DR, Krantz ID, TraegerSynodinos J. 2008. Mutations in the chromatin-associated protein ATRX. Hum Mutat 29: 796-802.

Goldberg AD, Banaszynski LA, Noh KM, Lewis PW, Elsaesser SJ, Stadler S, Dewell S, Law M, Guo X, Li X, et al. 2010. Distinct factors control histone variant H3.3 localization at specific genomic regions. Cell 140: 678691.

Gonzalez-Perez A, Perez-Llamas C, Deu-Pons J, Tamborero D, Schroeder MP, Jene-Sanz A, Santos A, Lopez-Bigas N. 2013. IntOGen-mutations identifies cancer drivers across tumor types. Nat Methods 10: 1081-1082.

Guerrini R, Shanahan JL, Carrozzo R, Bonanni P, Higgs DR, Gibbons RJ. 2000. A nonsense mutation of the ATRX gene causing mild mental retardation and epilepsy. Ann Neurol 47: 117-121.

He Q, Kim H, Huang R, Lu W, Tang M, Shi F, Yang D, Zhang X, Huang J, Liu D, et al. 2015. The Daxx/Atrx complex protects tandem repetitive elements during DNA hypomethylation by promoting H3K9 trimethylation. Cell Stem Cell 17: 273-286.

Heaphy CM, de Wilde RF, Jiao Y, Klein AP, Edil BH, Shi C, Bettegowda C, Rodriguez FJ, Eberhart CG, Hebbar S, et al. 2011. Altered telomeres in tumors with ATRX and DAXX mutations. Science 333: 425.

Helming KC, Wang X, Roberts CWM. 2014. Vulnerabilities of mutant SWI/SNF complexes in cancer. Cancer Cell 26: 309-317.

Hollenbach AD, Sublett JE, McPherson CJ, Grosveld G. 1999. The Pax3-FKHR oncoprotein is unresponsive to the Pax3-associated repressor hDaxx. EMBO J 18: $3702-3711$.

Hollenbach AD, McPherson CJ, Mientjes EJ, Iyengar R, Grosveld G. 2002. Daxx and histone deacetylase II associate with chromatin through an interaction with core histones and the chromatin-associated protein Dek. $J$ Cell Sci 115: 3319-3330.

Huh MS, Price O'Dea T, Ouazia D, McKay BC, Parise G, Parks RJ, Rudnicki MA, Picketts DJ. 2012. Compromised genomic integrity impedes muscle growth after Atrx inactivation. J Clin Invest 122: 4412-4423.

Huh MS, Ivanochko D, Hashem LE, Curtin M, Delorme M, Goodall E, Yan K, Picketts DJ. 2016. Stalled replication forks within heterochromatin require ATRX for protection. Cell Death Dis 7: e2220.

Huppert JL, Balasubramanian S. 2005. Prevalence of quadruplexes in the human genome. Nucleic Acids Res 33: 2908-2916.

Iwase S, Xiang B, Ghosh S, Ren T, Lewis PW, Cochrane JC, Allis CD, Picketts DJ, Patel DJ, Li H, et al. 2011. ATRX ADD domain links an atypical histone methylation recognition mechanism to human mental-retardation syndrome. Nat Struct Mol Biol 18: 769-776.

Jiao Y, Shi C, Edil BH, de Wilde RF, Klimstra DS, Maitra A, Schulick RD, Tang LH, Wolfgang CL, Choti MA, et al. 2011. DAXX/ATRX, MEN1, and mTOR pathway genes are frequently altered in pancreatic neuroendocrine tumors. Science 331: 1199-1203.

Johnson BE, Mazor T, Hong C, Barnes M, Aihara K, McLean CY, Fouse SD, Yamamoto S, Ueda H, Tatsuno K, et al. 2014. Mutational analysis reveals the origin and therapydriven evolution of recurrent glioma. Science 343: 189193.

Kannan K, Inagaki A, Silber J, Gorovets D, Zhang J, Kastenhuber ER, Heguy A, Petrini JH, Chan TA, Huse JT. 2012. Whole-exome sequencing identifies ATRX mutation as a key molecular determinant in lower-grade glioma. Oncotarget 3: 1194-1203.

Khuong-Quang DA, Buczkowicz P, Rakopoulos P, Liu XY, Fontebasso AM, Bouffet E, Bartels U, Albrecht S, Schwartzentruber J, Letourneau L, et al. 2012. K27M mutation in histone $\mathrm{H} 3.3$ defines clinically and biologically distinct subgroups of pediatric diffuse intrinsic pontine gliomas. Acta Neuropathol 124: 439-447.

Law MJ, Lower KM, Voon HPJ, Hughes JR, Garrick D, Viprakasit V, Mitson M, De Gobbi M, Marra M, Morris A, et al. 2010. ATR-X syndrome protein targets tandem repeats and influences allele-specific expression in a sizedependent manner. Cell 143: 367-378.

Lechner MS, Schultz DC, Negorev D, Maul GG, Rauscher FJ. 2005. The mammalian heterochromatin protein 1 binds diverse nuclear proteins through a common motif that targets the chromoshadow domain. Biochem Biophys Res Commun 331: 929-937.

Le Douarin B, Nielsen AL, Garnier JM, Ichinose H, Jeanmougin F, Losson R, Chambon P. 1996. A possible involvement of TIF $1 \alpha$ and TIF $1 \beta$ in the epigenetic control of transcription by nuclear receptors. EMBO J 15: 6701 6715.

Lee JC, Jeng YM, Liau JY, Tsai JH, Hsu HH, Yang CY. 2015. Alternative lengthening of telomeres and loss of ATRX are frequent events in pleomorphic and dedifferentiated liposarcomas. Mod Pathol 28: 1064-1073.

Leung JW-C, Ghosal G, Wang W, Shen X, Wang J, Li L, Chen J. 2013. $\alpha$-Thalassemia/mental retardation syndrome $\mathrm{X}$ linked gene product ATRX is required for proper replication restart and cellular resistance to replication stress. $J$ Biol Chem 288: 6342-6350.

Levy MA, Kernohan KD, Jiang Y, Bérubé NG. 2015. ATRX promotes gene expression by facilitating transcriptional elongation through guanine-rich coding regions. Hum Mol Genet 24: 1824-1835.

Lewis PW, Elsaesser SJ, Noh KM, Stadler SC, Allis CD. 2010. Daxx is an H3.3-specific histone chaperone and cooperates with ATRX in replication-independent chromatin 
assembly at telomeres. Proc Natl Acad Sci 107: 1407514080.

Lewis PW, Müller MM, Koletsky MS, Cordero F, Lin S, Banaszynski LA, Garcia BA, Muir TW, Becher OJ, Allis CD. 2013. Inhibition of PRC2 activity by a gain-of-function $\mathrm{H} 3$ mutation found in pediatric glioblastoma. Science 340: $857-861$.

Li R, Pei H, Watson DK, Papas TS. 2000. EAP1/Daxx interacts with ETS1 and represses transcriptional activation of ETS1 target genes. Oncogene 19: 745-753.

Liau JY, Lee JC, Tsai JH, Yang CY, Liu TL, Ke ZL, Hsu HH, Jeng YM. 2015a. Comprehensive screening of alternative lengthening of telomeres phenotype and loss of ATRX expression in sarcomas. Mod Pathol 28: 1545-1554.

Liau JY, Tsai JH, Jeng YM, Lee JC, Hsu HH, Yang CY. 2015b. Leiomyosarcoma with alternative lengthening of telomeres is associated with aggressive histologic features, loss of ATRX expression, and poor clinical outcome. Am J Surg Pathol 39: 236-244.

Liau JY, Tsai JH, Yang CY, Lee JC, Liang CW, Hsu HH, Jeng YM. 2015c. Alternative lengthening of telomeres phenotype in malignant vascular tumors is highly associated with loss of ATRX expression and is frequently observed in hepatic angiosarcomas. Hum Pathol 46: 1360-1366.

Liu CP, Xiong C, Wang M, Yu Z, Yang N, Chen P, Zhang Z, Li G, Xu R-M. 2012a. Structure of the variant histone H3.3H4 heterodimer in complex with its chaperone DAXX. Nat Struct Mol Biol 19: 1287-1292.

Liu XY, Gerges N, Korshunov A, Sabha N, Khuong-Quang DA, Fontebasso AM, Fleming A, Hadjadj D, Schwartzentruber J, Majewski J, et al. 2012b. Frequent ATRX mutations and loss of expression in adult diffuse astrocytic tumors carrying IDH1/IDH2 and TP53 mutations. Acta Neuropathol 124: 615-625.

Lovejoy CA, Li W, Reisenweber S, Thongthip S, Bruno J, de Lange T, De S, Petrini JHJ, Sung PA, Jasin M, et al. 2012. Loss of ATRX, genome instability, and an altered DNA damage response are hallmarks of the alternative lengthening of telomeres pathway. PLoS Genet 8: e1002772.

Lu C, Ward PS, Kapoor GS, Rohle D, Turcan S, Abdel-Wahab O, Edwards CR, Khanin R, Figueroa ME, Melnick A, et al. 2012. IDH mutation impairs histone demethylation and results in a block to cell differentiation. Nature 483: 474478.

McDowell TL, Gibbons RJ, Sutherland H, O’Rourke DM, Bickmore WA, Pombo A, Turley H, Gatter K, Picketts DJ, Buckle VJ, et al. 1999. Localization of a putative transcriptional regulator (ATRX) at pericentromeric heterochromatin and the short arms of acrocentric chromosomes. Proc Natl Acad Sci 96: 13983-13988.

Medina CF, Mazerolle C, Wang Y, Bérubé NG, Coupland S, Gibbons RJ, Wallace VA, Picketts DJ. 2009. Altered visual function and interneuron survival in Atrx knockout mice: Inference for the human syndrome. Hum $\mathrm{Mol} \mathrm{Ge}$ net 18: 966-977.

Michaelson JS, Bader D, Frank K, Kozak C, Leder P. 1999. Loss of Daxx, a promiscuously interacting protein, results in extensive apoptosis in early mouse development. Genes Dev 13: 1918-1923.

Mitson M, Kelley LA, Sternberg MJE, Higgs DR, Gibbons RJ. 2011. Functional significance of mutations in the Snf2 domain of ATRX. Hum Mol Genet 20: 2603-2610.
Molenaar JJ, Koster J, Zwijnenburg DA, van Sluis P, Valentijn LJ, van der Ploeg I, Hamdi M, van Nes J, Westerman BA, van Arkel J, et al. 2012. Sequencing of neuroblastoma identifies chromothripsis and defects in neuritogenesis genes. Nature 483: 589-593.

Nowick K, Fields C, Gernat T, Caetano-Anolles D, Kholina N, Stubbs L. 2011. Gain, loss and divergence in primate Zinc-Finger genes: A rich resource for evolution of gene regulatory differences between species. PLOS ONE 6: e21553.

Olar A, Sulman EP. 2015. Molecular markers in low-grade glioma-toward tumor reclassification. Semin Radiat Oncol 25: 155-163.

O'Sullivan RJ, Arnoult N, Lackner DH, Oganesian L, Haggblom C, Corpet A, Almouzni G, Karlseder J. 2014. Rapid induction of alternative lengthening of telomeres by depletion of the histone chaperone ASF1. Nat Struct Mol Biol 21: 167-174.

Peifer M, Hertwig F, Roels F, Dreidax D, Gartlgruber M, Menon R, Krämer A, Roncaioli JL, Sand F, Heuckmann JM, et al. 2015. Telomerase activation by genomic rearrangements in high-risk neuroblastoma. Nature 526: 700-704.

Perrem K, Bryan TM, Englezou A, Hackl T, Moy EL, Reddel RR. 1999. Repression of an alternative mechanism for lengthening of telomeres in somatic cell hybrids. Oncogene 18: 3383-3390.

Pinto EM, Chen X, Easton J, Finkelstein D, Liu Z, Pounds S, Rodriguez-Galindo C, Lund TC, Mardis ER, Wilson RK, et al. 2015. Genomic landscape of paediatric adrenocortical tumours. Nat Commun 6: 6302.

Pugh TJ, Morozova O, Attiyeh EF, Asgharzadeh S, Wei JS, Auclair D, Carter SL, Cibulskis K, Hanna M, Kiezun A, et al. 2013. The genetic landscape of high-risk neuroblastoma. Nat Genet 45: 279-284.

Qadeer ZA, Harcharik S, Valle-Garcia D, Chen C, Birge MB, Vardabasso C, Duarte LF, Bernstein E. 2014. Decreased expression of the chromatin remodeler ATRX associates with melanoma progression. J Invest Dermatol 134: $1768-1772$.

Ramamoorthy M, Smith S. 2015. Loss of ATRX suppresses resolution of telomere cohesion to control recombination in ALT cancer cells. Cancer Cell 28: 357-369.

Ratnakumar K, Duarte LF, LeRoy G, Hasson D, Smeets D, Vardabasso C, Bonisch C, Zeng T, Xiang B, Zhang DY, et al. 2012. ATRX-mediated chromatin association of histone variant macroH2A1 regulates $\alpha$-globin expression. Genes Dev 26: 433-438.

Rosnoblet C, Vandamme J, Völkel P, Angrand P-O. 2011. Analysis of the human HP1 interactome reveals novel binding partners. Biochem Biophys Res Commun 413: 206-211.

Sadic D, Schmidt K, Groh S, Kondofersky I, Ellwart J, Fuchs C, Theis FJ, Schotta G. 2015. Atrx promotes heterochromatin formation at retrotransposons. EMBO Rep 16: $836-850$.

Sarma K, Cifuentes-Rojas C, Ergun A, Del Rosario A, Jeon Y, White F, Sadreyev R, Lee JT. 2014. ATRX directs binding of PRC2 to Xist RNA and Polycomb targets. Cell 159: 869-883.

Schwartzentruber J, Korshunov A, Liu X-Y, Jones DTW, Pfaff E, Jacob K, Sturm D, Fontebasso AM, Quang D- 
M.A. Dyer et al.

AK, Tönjes M, et al. 2012. Driver mutations in histone H3.3 and chromatin remodelling genes in paediatric glioblastoma. Nature 482: 226-231.

Solomon LA, Russell BA, Watson LA, Beier F, Bérubé NG. 2013. Targeted loss of the ATR-X syndrome protein in the limb mesenchyme of mice causes brachydactyly. Hum Mol Genet 22: 5015-5025.

Tagami H, Ray-Gallet D, Almouzni G, Nakatani Y. 2004. Histone H3.1 and H3.3 complexes mediate nucleosome assembly pathways dependent or independent of DNA synthesis. Cell 116: 51-61.

Tang J, Wu S, Liu H, Stratt R, Barak OG, Shiekhattar R, Picketts DJ, Yang X. 2004. A novel transcription regulatory complex containing death domain-associated protein and the ATR-X syndrome protein. J Biol Chem 279: 20369-20377.

TCGA. 2015. The Cancer Genome Atlas, cancergenome .nih.gov.

Torii S, Egan DA, Evans RA, Reed JC. 1999. Human Daxx regulates Fas-induced apoptosis from nuclear PML oncogenic domains (PODs). EMBO J 18: 6037-6049.

Turcan S, Rohle D, Goenka A, Walsh LA, Fang F, Yilmaz E, Campos C, Fabius AWM, Lu C, Ward PS, et al. 2012. IDH1 mutation is sufficient to establish the glioma hypermethylator phenotype. Nature 483: 479-483.

Valentijn LJ, Koster J, Zwijnenburg DA, Hasselt NE, van Sluis P, Volckmann R, van Noesel MM, George RE, Tytgat GAM, Molenaar JJ, et al. 2015. TERT rearrangements are frequent in neuroblastoma and identify aggressive tumors. Nat Genet 47: 1411-1414.

Valle-Garcia D, Qadeer ZA, McHugh D, Ghiraldini FG, Chowdhury AH, Hasson D, Dyer MA, Recillas-Targa F, Bernstein E. 2016. ATRX binds to atypical chromatin domains at the $3^{\prime}$ exons of ZNF genes to pre serve H3K9me3 enrichment. Epigenetics doi: 10.1080/ 15592294.2016.1169351.
Voon HPJ, Hughes JR, Rode C, DeLaRosa-Velazquez IA, Jenuwein T, Feil R, Higgs DR, Gibbons RJ. 2015. ATRX plays a key role in maintaining silencing at interstitial heterochromatic loci and imprinted genes. Cell Rep 11: 405-418.

Watson LA, Solomon LA, Li JR, Jiang Y, Edwards M, Shin-Ya K, Beier F, Berube NG. 2013. Atrx deficiency induces telomere dysfunction, endocrine defects, and reduced life span. J Clin Invest 123: 2049-2063.

Watson LA, Goldberg H, Bérubé NG. 2015. Emerging roles of ATRX in cancer. Epigenomics 7: 1365-1378.

Wong LH. 2010. Epigenetic regulation of telomere chromatin integrity in pluripotent embryonic stem cells. Epigenomics 2: 639-655.

Wu G, Broniscer A, McEachron TA, Lu C, Paugh BS, Becksfort J, Qu C, Ding L, Huether R, Parker M, et al. 2012. Somatic histone $\mathrm{H} 3$ alterations in pediatric diffuse intrinsic pontine gliomas and non-brainstem glioblastomas. Nat Genet 44: 251-253.

Wu G, Diaz AK, Paugh BS, Rankin SL, Ju B, Li Y, Zhu X, Qu C, Chen X, Zhang J, et al. 2014. The genomic landscape of diffuse intrinsic pontine glioma and pediatric non-brainstem high-grade glioma. Nat Genet 46: 444-450.

Xue Y, Gibbons R, Yan Z, Yang D, McDowell TL, Sechi S, Qin J, Zhou S, Higgs D, Wang W. 2003. The ATRX syndrome protein forms a chromatin-remodeling complex with Daxx and localizes in promyelocytic leukemia nuclear bodies. Proc Natl Acad Sci 100: 10635-10640.

Yang X, Khosravi-Far R, Chang HY, Baltimore D. 1997. Daxx, a novel Fas-binding protein that activates JNK and apoptosis. Cell 89: 1067-1076.

Zhong S, Salomoni P, Ronchetti S, Guo A, Ruggero D, Pandolfi PP. 2000. Promyelocytic leukemia protein (PML) and Daxx participate in a novel nuclear pathway for apoptosis. J Exp Med 191: 631-640. 


\section{$\&_{\mathrm{CSH}}^{\infty} \&$ Cold Spring Harbor

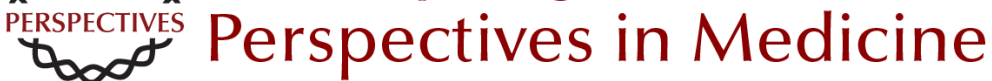

\section{ATRX and DAXX: Mechanisms and Mutations}

Michael A. Dyer, Zulekha A. Qadeer, David Valle-Garcia and Emily Bernstein

Cold Spring Harb Perspect Med 2017; doi: 10.1101/cshperspect.a026567 originally published online January 6, 2017

\section{Subject Collection Chromatin Deregulation in Cancer}

\section{Mixed-Lineage Leukemia Fusions and Chromatin in Leukemia \\ Andrei V. Krivtsov, Takayuki Hoshii and Scott A. Armstrong}

Targeting Cancer Cells with BET Bromodomain Inhibitors

Yali Xu and Christopher R. Vakoc

The Role of Nuclear Receptor-Binding SET

Domain Family Histone Lysine Methyltransferases

in Cancer

Richard L. Bennett, Alok Swaroop, Catalina Troche, et al.

SETting the Stage for Cancer Development:

SETD2 and the Consequences of Lost Methylation Catherine C. Fahey and lan J. Davis

ATRX and DAXX: Mechanisms and Mutations Michael A. Dyer, Zulekha A. Qadeer, David Valle-Garcia, et al.

DNMT3A in Leukemia

Lorenzo Brunetti, Michael C. Gundry and Margaret A. Goodell

Oncogenic Mechanisms of Histone H3 Mutations Daniel N. Weinberg, C. David Allis and Chao Lu

Nonhistone Lysine Methylation in the Regulation of Cancer Pathways

Scott M. Carlson and Or Gozani
TET2 in Normal and Malignant Hematopoiesis Robert L. Bowman and Ross L. Levine

Long Noncoding RNAs: At the Intersection of Cancer and Chromatin Biology Adam M. Schmitt and Howard Y. Chang

DNA Hypomethylating Drugs in Cancer Therapy Takahiro Sato, Jean-Pierre J. Issa and Patricia Kropf

The Chromodomain Helicase DNA-Binding Chromatin Remodelers: Family Traits that Protect from and Promote Cancer Alea A. Mills

Exploitation of EP300 and CREBBP Lysine Acetyltransferases by Cancer Narsis Attar and Siavash K. Kurdistani

Histone Lysine Demethylase Inhibitors Ashwini Jambhekar, Jamie N. Anastas and Yang Shi

Cohesin Mutations in Cancer Magali De Koninck and Ana Losada

MLL3/MLL4/COMPASS Family on Epigenetic Regulation of Enhancer Function and Cancer Christie C. Sze and Ali Shilatifard

For additional articles in this collection, see http://perspectivesinmedicine.cshlp.org/cgi/collection/ 